\title{
The Association of Overt and Subclinical Hyperthyroidism with the Risk of Cardiovascular Events and Cardiovascular Mortality: Meta-Analysis and Systematic Review of Cohort Studies
}

\author{
Seo Young Sohn ${ }^{1}$, Eunyoung Lee ${ }^{2}$, Min Kyung Lee ${ }^{1}$, Jae Hyuk Lee ${ }^{1}$ \\ ${ }^{1}$ Division of Endocrinology and Metabolism, Department of Internal Medicine, Myongji Hospital, Hanyang University College \\ of Medicine, Goyang; ${ }^{2}$ Office of Biostatistics, Medical Research Collaboration Center, Ajou Research Institute for Innovative \\ Medicine, Ajou University Medical Center, Seoul, Korea
}

Background: Whether hyperthyroidism is an independent risk factor for cardiovascular events remains controversial. We aimed to evaluate the association of overt and subclinical hyperthyroidism with the risk of ischemic heart disease (IHD), stroke, heart failure, and cardiovascular mortality.

Methods: Studies regarding the association between hyperthyroidism and cardiovascular events were searched on PubMed and Embase databases. The cardiovascular disease (CVD) risk was classified as high and low, based on pre-existing diseases, including history of coronary, cerebral, or peripheral artery disease; heart failure; atrial fibrillation; diabetes mellitus; or chronic kidney disease.

Results: Thirty-seven cohort studies were included in this meta-analysis. The pooled hazard ratio for subjects with overt hyperthyroidism compared with the control group was 1.11 (95\% confidence interval [CI], 1.03 to 1.19) for IHD, 1.35 (95\% CI, 1.03 to 1.75) for stroke, and 1.20 (95\% CI, 1.00 to 1.46) for cardiovascular mortality. For subjects with subclinical hyperthyroidism, the pooled hazard ratio was 1.24 (95\% CI, 1.07 to 1.45) for IHD, when compared with the control group. Subgroup analysis by CVD risk showed that the risk of stroke in overt hyperthyroidism was increased in the low CVD risk group; however, these association was not observed in the high CVD risk group. Similarly, the risk of IHD in subjects with subclinical hyperthyroidism was significantly increased in the low CVD risk group.

Conclusion: Overt hyperthyroidism is associated with increased risk of IHD, stroke, and cardiovascular mortality, and subclinical hyperthyroidism is associated with increased risk of IHD. These associations were particularly observed in the low risk CVD group without underlying CVD.

Keywords: Hyperthyroidism; Myocardial infarction; Stroke; Heart failure; Mortality; Meta-analysis

\section{INTRODUCTION}

Hyperthyroidismw is a common endocrine disease, with a reported prevalence of $0.3 \%$ to $3 \%$, depending on the age, sex, and iodine intake [1]. Hyperthyroidism is known to have an impact on the cardiovascular (CV) system, such as increased heart rate, contractility, systolic hypertension, changes in peripheral vascular resistance, atrial fibrillation (AF), and hypercoagulabil-
Received: 3 June 2020, Revised: 8 August 2020, Accepted: 21 September 2020 Corresponding author: Seo Young Sohn

Division of Endocrinology and Metabolism, Department of Internal Medicine, Myongji Hospital, Hanyang University College of Medicine, 55 Hwasu-ro 14beon-gil, Deokyang-gu, Goyang 10475, Korea Tel: +82-31-810-5409, Fax: +82-31-969-0500, E-mail: drsohnsy@gmail.com
Copyright $\odot 2020$ Korean Endocrine Society

This is an Open Access article distributed under the terms of the Creative Commons Attribution Non-Commercial License (https://creativecommons.org/ licenses/by-nc/4.0/) which permits unrestricted non-commercial use, distribution, and reproduction in any medium, provided the original work is properly cited. 
ity $[2,3]$. As a milder form of hyperthyroidism, subclinical hyperthyroidism is also associated with systolic hypertension, AF, and diastolic dysfunction, especially when the thyroid stimulating hormone (TSH) level is less than $0.1 \mathrm{mIU} / \mathrm{L}[2,4]$. Although both overt hyperthyroidism and subclinical hyperthyroidism are associated with various CV risk factors, whether they are independent risk factors for $\mathrm{CV}$ events or $\mathrm{CV}$ mortality remains controversial [2,3,5-46].

Several meta-analyses, including individual participant data meta-analyses, investigating the relationship of hyperthyroidism with CV events and/or CV mortality have been published; however, most of them have been limited because they were restricted to patients with subclinical hyperthyroidism and provided conflicting results $[12,14,19]$. Furthermore, it remains unclear whether the risk of cardiovascular disease (CVD) in hyperthyroidism varies by age and individual CVD risk. Many additional cohort studies have been published recently $[9,17,20,21,25-29$, $32,33,42]$. Therefore, an updated comprehensive meta-analysis is warranted to clarify the association of hyperthyroidism with CV events.

\section{METHODS}

We conducted meta-analysis and the systematic review based on a predefined protocol (Appendix 1) and reported the results based on the Preferred Reporting Items for Systematic Review and Meta-Analysis (PRISMA) statement (Supplemental Table S1).

\section{Search strategy}

A literature search was performed on the PubMed and Embase databases from their inception (January 1, 1990) to February 28, 2020. The reference list of recent reviews, related articles, and meta-analyses were also reviewed. The search strategy was conducted by medical subject headings ("hyperthyroidism," "thyroid diseases," "thyroid hormones," "myocardial ischemia," "heart failure," "stroke," "brain ischemia," "mortality," and "cardiovascular disease") and with text words ("subclinical thyroid," "thyroid diseases," "coronary heart disease," and "ischemic heart disease"). We also searched for grey literature from the website of OpenGrey [47]. The specific searching strategy is described in the Appendix 1. Only articles published in English were considered.

\section{Study selection}

Two independent reviewers (S.Y.S. and E.L.) initially screened the titles and abstracts. From this, studies that were clearly irrel- evant were excluded. Any disagreements were resolved by a third reviewer (M.K.L.).

A second screening was based on full-text reviews. Studies were included if they met the following criteria: (1) the study design was a cohort study; (2) the outcome of interest was considered as ischemic heart disease (IHD), stroke, heart failure, or CV mortality; (3) the study compared the endogenous hyperthyroidism between the euthyroid control group and the general population without hyperthyroidism; and (4) the effect measure was reported as hazard ratio (HR) with $95 \%$ confidence interval (CI). The following studies were excluded: (1) the study design was not a cohort study; (2) the outcome of interest was not related to a CVD; (3) the study included duplicated datasets; (4) the study investigated the effect of exogenous hyperthyroidism; and (5) the study had insufficient data.

\section{Data extraction and quality assessment}

Two reviewers independently extracted the data using a standardized data collection form. The following data were extracted from the included articles: first author's name, publication year, country, number of study participants, characteristics of study participants, mean age, and CV outcome explored.

The Newcastle-Ottawa Scale - a validated tool for non-randomized studies in meta-analysis - was used to evaluate the methodological quality of the included studies. Reviewers independently assessed the selection of studies, comparability, and their outcomes using a rating system - low, moderate, and high risk - depending on the scoring for each section as presented in Newcastle-Ottawa Scale assessment. Any disagreements were resolved by a third investigator.

\section{Statistical analyses}

The study-specific maximally adjusted HR was used to compute a summary HR and its $95 \%$ CI. Heterogeneity was graded using the $I^{2}$ statistics, with $I^{2}$ of less than $25 \%$, in between $25 \%$ and $50 \%$, and greater than $50 \%$ representing low, moderated and high heterogeneity, respectively. The Higgins' $I^{2}$ statistic was used to test for heterogeneity. When $I^{2}$ was $\leq 50 \%$, the included studies were considered to have little heterogeneity, and a fixed-effects model was used. When $I^{2}$ was $>50 \%$, the included studies were considered to have significant heterogeneity, and a random effects model was used.

We performed sensitivity and subgroup analyses to explore and interpret the sources of high heterogeneity. We explored the relationship between hyperthyroidism and $\mathrm{CV}$ outcomes by age ( $<65$ or $\geq 65$ years), pre-existing CVD and regional iodine in- 
take status (sufficient areas vs. deficient areas) [48]. The high CVD risk group was defined as those with any underlying disease that could increase the risk of CVD, including history of coronary, cerebral, or peripheral artery disease; dilated cardiomyopathy; heart failure; AF; diabetes mellitus; or chronic kidney disease. The low CVD risk group was defined as those without diseases that would increase the risk of CVD. All statistical analyses were performed using Review Manager version 5.3 (https://training.cochrane.org).

\section{Ethical approval}

This article does not contain examinations performed on human participants in that ethical approval is not necessary.

\section{RESULTS}

\section{Characteristics of included studies}

A study selection flow chart is presented in Fig. 1. A total of 15,118 studies were initially identified, and 13,075 remained after excluding duplicate citations. Of these, most studies were excluded after the first screening based on the abstracts or titles, mainly because they were reviews, case reports, conference abstracts, meta-analyses or different topic of interest. After a full text review of 143 studies, 106 studies were further excluded, and 37 studies [5-11,13,15-17,20-44,46] were finally included for analysis. No additional studies which met the inclusion criteria were found on OpenGrey (http://www.opengrey.eu).

Characteristics of the studies are presented in Table 1. In total, 1,626,005 participants were enrolled, and 113,393 had hyperthyroidism. Sample sizes of these studies ranged from 269 to $1,239,441$ participants. Among them, 22 studies showed a mean age of $<65$ years, and 15 had a mean age of $\geq 65$ years. A total of 11 studies were defined as involving participants at high CVD risk based on pre-existing diseases. Twenty-six studies were population-based studies with participants from a general population. They were classified as low CVD risk. All included studies were judged as being of relatively high quality according to the Newcastle-Ottawa Scale assessment tool, with scores ranging from 6 to 9 (Supplemental Table S2).

\section{Hyperthyroidism and Ischemic heart disease}

Four eligible studies on the association between overt hyperthyroidism and IHD were pooled (Fig. 2A). We found that overt hyperthyroidism was related to IHD (HR, 1.11; 95\% CI, 1.03 to $1.19)$ with evidence of low heterogeneity $\left(I^{2}=0 \%, P=0.70\right)$. Because, all eligible studies included patients with age younger than 65 years and low CVD risk, subgroup analysis by age and CVD risk was not available (Table 2). In the subgroup analysis

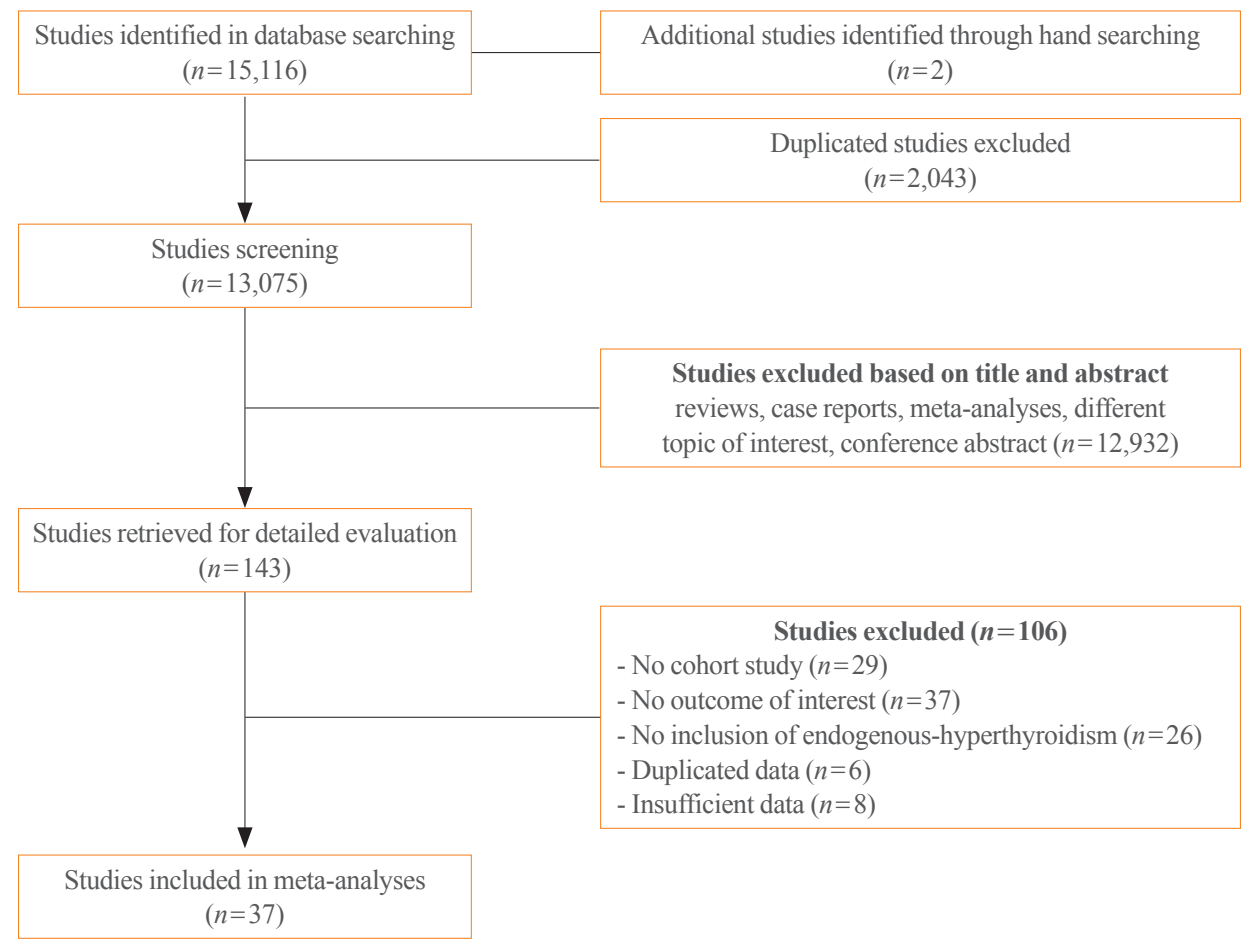

Fig. 1. Flowchart of study screening and selection. 
Table 1. Baseline Characteristics of Cohort Studies Included in the Present Meta-Analysis

\begin{tabular}{|c|c|c|c|c|c|c|c|}
\hline Study & Country & Study population & $\begin{array}{l}\text { Classification } \\
\text { of hyperthy- } \\
\text { roidism }\end{array}$ & Mean or median age & $\begin{array}{l}\text { No. of total } \\
\text { subjects }\end{array}$ & $\begin{array}{l}\text { No. of hyper- } \\
\text { thyroid } \\
\text { subjects }\end{array}$ & CV outcome \\
\hline $\begin{array}{l}\text { Kim et al. (2020) } \\
{[26]}\end{array}$ & Korea & Community dwelling & Overt & $\begin{array}{l}\text { Overt: } 48.5 \\
\text { Control: } 48.5\end{array}$ & $1,239,441$ & 59,021 & IHD and stroke \\
\hline $\begin{array}{l}\text { Okosieme et al. } \\
\text { (2019) [32] }\end{array}$ & England & Community dwelling & Overt & $\begin{array}{l}\text { Overt: } 48 \pm 16 \\
\text { Control: } 48 \pm 16\end{array}$ & 20,945 & 4,189 & $\begin{array}{l}\text { IHD, stroke, } \\
\text { and HF }\end{array}$ \\
\hline $\begin{array}{l}\text { Langen et al. (2018) } \\
\text { [28] }\end{array}$ & Finland & Community dwelling & Subclinical & $\begin{array}{l}\text { Subclinical: } 56.8 \pm 16.5 \\
\text { Control: } 50.5 \pm 13.8\end{array}$ & 5,211 & 108 & IHD and stroke \\
\hline $\begin{array}{l}\text { Ryodi et al. (2018) } \\
\text { [17] }\end{array}$ & Finland & Community dwelling & Overt & $49(35-63)$ & 24,580 & 6,148 & $\begin{array}{l}\text { IHD, stroke, } \\
\text { HF, and CV } \\
\text { mortality }\end{array}$ \\
\hline $\begin{array}{l}\text { Journy et al. (2017) } \\
\text { [25] }\end{array}$ & United States & Community dwelling & Overt & $\begin{array}{l}\text { Overt: }<60 \text { years } \\
\text { Control: }<60 \text { years }\end{array}$ & 75,076 & 1,501 & CV mortality \\
\hline $\begin{array}{l}\text { Giesecke et al. } \\
\text { (2017) [21] }\end{array}$ & Sweden & Community dwelling & Overt & $\begin{array}{l}\text { Overt: } 61.3 \\
\text { Control: } 49.1\end{array}$ & 15,924 & 12,239 & CV mortality \\
\hline $\begin{array}{l}\text { Martin et al. (2017) } \\
\text { [29] }\end{array}$ & United States & Community dwelling & $\begin{array}{l}\text { Overt, subclini- } \\
\text { cal }\end{array}$ & $\begin{array}{l}\text { Overt: } 56.7 \pm 5.8 \\
\text { Subclinical: } 56.2 \pm 5.7 \\
\text { Control: } 56.5 \pm 5.7\end{array}$ & 11,359 & $\begin{array}{l}\text { Overt: } 206 \\
\text { Subclinical: } \\
\quad 378\end{array}$ & IHD and stroke \\
\hline $\begin{array}{l}\text { Pearce et al. (2016) } \\
\text { [33] }\end{array}$ & $\begin{array}{l}\text { United } \\
\text { Kingdom }\end{array}$ & Community dwelling & Subclinical & $85.5 \pm 0.4$ & 643 & 19 & CV mortality \\
\hline $\begin{array}{l}\text { Bruere et al. (2015) } \\
\text { [9] }\end{array}$ & France & $\begin{array}{l}\text { High CVD risk } \\
\text { (A-fib patients) }\end{array}$ & Overt & $\begin{array}{l}\text { Overt: } 71 \pm 14 \\
\text { Control: } 68 \pm 13\end{array}$ & 8,962 & 141 & Stroke \\
\hline $\begin{array}{l}\text { Geng et al. (2015) } \\
{[20]}\end{array}$ & China & $\begin{array}{l}\text { High CVD risk } \\
\text { (type } 2 \text { diabetes) }\end{array}$ & Subclinical & $\begin{array}{l}\text { Subclinical: } 57.5 \pm 14.7 \\
\text { Control: } 58.8 \pm 13.5\end{array}$ & 1,115 & 74 & IHD \\
\hline $\begin{array}{l}\text { la Cour et al. (2015) } \\
\text { [27] }\end{array}$ & Denmark & Community dwelling & Overt & 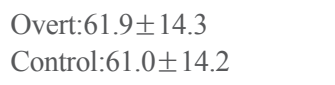 & 25,562 & 4,000 & Stroke \\
\hline $\begin{array}{l}\text { Yang et al. (2015) } \\
\text { [42] }\end{array}$ & Taiwan & Community dwelling & Overt & $\begin{array}{l}\text { Overt: } 40.9 \pm 14.3 \\
\text { Control: } 41.3 \pm 14.5\end{array}$ & 68,462 & 16,808 & Stroke \\
\hline $\begin{array}{l}\text { Chen et al. (2014) } \\
\text { [13] }\end{array}$ & China & $\begin{array}{l}\text { High CVD risk } \\
\text { (A-fib patients) }\end{array}$ & Overt & $\begin{array}{l}\text { Overt: } 55.1 \pm 1.7 \\
\text { Control: } 59.4 \pm 1.1\end{array}$ & 269 & 62 & Stroke \\
\hline $\begin{array}{l}\text { Drechsler et al. } \\
\text { (2014) [16] }\end{array}$ & Germany & $\begin{array}{l}\text { High CVD risk } \\
\text { (diabetic hemodialysis) }\end{array}$ & Subclinical & $\begin{array}{l}\text { Subclinical: } 66.9 \pm 7.9 \\
\text { Control: } 65.3 \pm 8.5\end{array}$ & 1,000 & 137 & IHD and stroke \\
\hline $\begin{array}{l}\text { Perez et al. (2014) } \\
\text { [34] }\end{array}$ & $\begin{array}{l}\text { Europe, } \\
\text { multicenter }\end{array}$ & $\begin{array}{r}\text { High CVD risk } \\
\text { (heart failure) }\end{array}$ & Subclinical & $\begin{array}{l}\text { Subclinical: } 72.9 \pm 6.2 \\
\text { Control: } 72.6 \pm 7.1\end{array}$ & 4,987 & 175 & $\begin{array}{l}\mathrm{HF} \text { and CV } \\
\text { mortality }\end{array}$ \\
\hline $\begin{array}{l}\text { Brandt et al. (2013) } \\
\text { [8] }\end{array}$ & Denmark & Community dwelling & Overt & $\begin{array}{l}\text { Graves' disease: } 55 \text { (18-96) } \\
\text { Toxic nodular goiter: } \\
\quad 62(18-96)\end{array}$ & 10,760 & 2,152 & CV mortality \\
\hline $\begin{array}{l}\text { Ceresini et al. } \\
\text { (2013) [11] }\end{array}$ & Italy & Community dwelling & Subclinical & $>65$ years & 951 & 83 & CV mortality \\
\hline $\begin{array}{l}\text { Asvold et al. (2012) } \\
\text { [5] }\end{array}$ & Norway & Community dwelling & Subclinical & $\begin{array}{l}\text { Male: } 53(46-65) \\
\text { Female: } 54(47-67)\end{array}$ & 26,707 & 524 & IHD and $\mathrm{HF}$ \\
\hline $\begin{array}{l}\text { Nanchen et al. } \\
\text { (2012) [31] }\end{array}$ & $\begin{array}{l}\text { Europe, } \\
\text { multicenter }\end{array}$ & $\begin{array}{l}\text { High CVD risk } \\
\text { (CVD patients) }\end{array}$ & Subclinical & $\begin{array}{l}\text { Subclinical: } 75.3 \pm 3.1 \\
\text { Control: } 75.3 \pm 3.4\end{array}$ & 5,316 & 71 & $\begin{array}{l}\text { IHD, HF, and } \\
\text { CV mortality }\end{array}$ \\
\hline $\begin{array}{l}\text { Waring et al. (2012) } \\
\text { [41] }\end{array}$ & United States & Community dwelling & Subclinical & $\begin{array}{l}\text { Subclinical: } 74.1 \\
\text { Control: } 73.6\end{array}$ & 1,587 & 41 & CV mortality \\
\hline $\begin{array}{l}\text { Molinaro et al. } \\
\text { (2012) [30] }\end{array}$ & Italy & High CVD risk & Subclinical & $\begin{array}{l}\text { Subclinical: } 69.3(65-73) \\
\text { Control: } 65(64-66)\end{array}$ & 1,026 & 23 & CV mortality \\
\hline
\end{tabular}




\begin{tabular}{|c|c|c|c|c|c|c|c|}
\hline Study & Country & Study population & $\begin{array}{l}\text { Classification } \\
\text { of hyperthy- } \\
\text { roidism }\end{array}$ & Mean or median age & $\begin{array}{l}\text { No. of total } \\
\text { subjects }\end{array}$ & $\begin{array}{c}\text { No. of hyper- } \\
\text { thyroid } \\
\text { subjects }\end{array}$ & CV outcome \\
\hline $\begin{array}{l}\text { de Jongh et al. } \\
\text { (2011) [15] }\end{array}$ & Netherland & Community dwelling & Subclinical & $\begin{array}{l}\text { Subclinical: } 77.7 \pm 7.0 \\
\text { Control: } 75.5 \pm 6.5\end{array}$ & 1,219 & 34 & CV mortality \\
\hline $\begin{array}{l}\text { Schultz et al. (2011) } \\
\text { [36] }\end{array}$ & Denmark & $\begin{array}{l}\text { High CVD risk } \\
\text { (type } 2 \text { diabetes) }\end{array}$ & Subclinical & $\begin{array}{l}\text { Subclinical: } 74 \pm 10 \\
\text { Control: } 67.5 \pm 10.5\end{array}$ & 609 & 25 & $\begin{array}{l}\text { Stroke and CV } \\
\text { mortality }\end{array}$ \\
\hline $\begin{array}{l}\text { Boekholdt et al. } \\
\text { (2010) [7] }\end{array}$ & $\begin{array}{l}\text { United } \\
\text { Kingdom }\end{array}$ & Community dwelling & Subclinical & $\begin{array}{l}\text { Subclinical: } 59 \pm 10 \\
\text { Control: } 58 \pm 9\end{array}$ & 11,554 & 216 & IHD \\
\hline $\begin{array}{l}\text { Ittermann et al. } \\
\text { (2010) [24] }\end{array}$ & German & Community dwelling & $\begin{array}{l}\text { Overt, } \\
\text { subclinical }\end{array}$ & $\begin{array}{l}\text { Subclinical: } 61 \text { (48-69) } \\
\text { Overt: NA } \\
\text { Control: } 48 \text { (35-62) }\end{array}$ & 3,651 & $\begin{array}{l}\text { Subclinical: } \\
243 \\
\text { Overt: } 27\end{array}$ & CV mortality \\
\hline $\begin{array}{l}\text { Sheu et al. (2010) } \\
\text { [38] }\end{array}$ & Taiwan & Community dwelling & Overt & $\begin{array}{l}\text { Overt: } 32.1 \pm 7.4 \\
\text { Control: } 32.1 \pm 7.5\end{array}$ & 28,584 & 3,176 & Stroke \\
\hline $\begin{array}{l}\text { Sgarbi et al. (2010) } \\
\text { [37] }\end{array}$ & Brazil & Community dwelling & Subclinical & $\begin{array}{l}\text { Subclinical: } 61.4 \pm 12.5 \\
\text { Control: } 56.4 \pm 12.4\end{array}$ & 1,110 & 69 & CV mortality \\
\hline $\begin{array}{l}\text { Siu et al. (2009) } \\
\text { [39] }\end{array}$ & Hong Kong & $\begin{array}{l}\text { High CVD risk } \\
\text { (A-fib patients) }\end{array}$ & Overt & $\begin{array}{l}\text { Overt: } 64.7 \pm 1.3 \\
\text { Control: } 64.7 \pm 1.1\end{array}$ & 480 & 160 & Stroke \\
\hline $\begin{array}{l}\text { Volzke et al. (2009) } \\
\text { [40] }\end{array}$ & Germany & $\begin{array}{l}\text { High CVD risk } \\
\text { (IHD patients) }\end{array}$ & Subclinical & $\begin{array}{l}\text { Subclinical: } 62.0 \pm 7.9 \\
\text { Control: } 61.0 \pm 8.0\end{array}$ & 942 & 118 & CV mortality \\
\hline $\begin{array}{l}\text { Rodondi et al. } \\
\text { (2008) [35] }\end{array}$ & United States & Community dwelling & Subclinical & $\begin{array}{l}\text { Subclinical: } 73.8 \pm 6.9 \\
\text { Control: } 72.5 \pm 5.5\end{array}$ & 3,044 & 44 & $\mathrm{HF}$ \\
\hline $\begin{array}{l}\text { Bauer et al. (2007) } \\
\text { [6] }\end{array}$ & United States & Community dwelling & Overt & $\begin{array}{l}\text { Overt: } 72.3 \pm 5.6 \\
\text { Control: } 71.6 \pm 5.3\end{array}$ & 9,449 & 891 & CV mortality \\
\hline $\begin{array}{l}\text { Iervasi et al. (2007) } \\
\text { [23] }\end{array}$ & Italy & $\begin{array}{l}\text { High CVD } \\
\text { (IHD patients) }\end{array}$ & Subclinical & $\begin{array}{l}\text { Subclinical: } 60.5(59-62) \\
\text { Control: } 59.9(59-60)\end{array}$ & 3,121 & 98 & $\begin{array}{l}\text { CV mortality, } \\
\text { IHD }\end{array}$ \\
\hline $\begin{array}{l}\text { Cappola et al. } \\
\text { (2006) [10] }\end{array}$ & United States & Community dwelling & Subclinical & $\begin{array}{l}\text { Subclinical: } 73.9 \pm 6.8 \\
\text { Control: } 72.6 \pm 5.6\end{array}$ & 3,233 & 31 & $\begin{array}{l}\text { IHD, stroke, } \\
\text { and CV } \\
\text { mortality }\end{array}$ \\
\hline $\begin{array}{l}\text { Qureshi et al. (2006) } \\
\text { [44] }\end{array}$ & United States & Community dwelling & Overt & $48 \pm 14$ & 5,269 & 34 & Stroke \\
\hline $\begin{array}{l}\text { Walsh et al. (2005) } \\
\text { [46] }\end{array}$ & Australia & Community dwelling & Subclinical & $\begin{array}{l}\text { Subclinical: } 51.3 \pm 14.9 \\
\text { Control: } 49.2 \pm 17.0\end{array}$ & 2,108 & 37 & $\begin{array}{l}\text { IHD and CV } \\
\text { mortality }\end{array}$ \\
\hline $\begin{array}{l}\text { Gussekloo et al. } \\
\text { (2004) [22] }\end{array}$ & Netherland & Community dwelling & Subclinical & $>85$ years & 558 & 19 & $\begin{array}{l}\text { CV mortality, } \\
\text { IHD }\end{array}$ \\
\hline $\begin{array}{l}\text { Parle et al. (2001) } \\
\text { [43] }\end{array}$ & $\begin{array}{l}\text { United King- } \\
\text { dom }\end{array}$ & Community dwelling & Subclinical & $\begin{array}{l}\text { Male } 70.1(69.6-70.6) \\
\text { Female } 70.7(70.2-71.4)\end{array}$ & 1,191 & 71 & CV mortality \\
\hline
\end{tabular}

CV, cardiovascular; IHD, ischemic heart disease; HF, heart failure; CVD, cardiovascular disease; NA, not available; A-fib, atrial fibrillation.

by iodine intake status, increased risk of IHD was observed in iodine sufficient areas (Supplemental Table S3).

For subclinical hyperthyroidism, 11 eligible studies were pooled to calculate the overall HR for IHD (Fig. 2B). Compared with euthyroid subjects, patients with subclinical hyperthyroidism was associated with IHD (HR, 1.24; 95\% CI, 1.07 to 1.45 ) and the heterogeneity was low $\left(I^{2}=0 \%, P=0.79\right)$. In the subgroup analysis by age ( $<65$ or $\geq 65$ years) and CVD risk, increased risk of IHD was found among subjects aged less than 65 years
(HR, 1.27; 95\% CI, 1.06 to 1.53 ) and in those with low CVD risk (HR, 1.22; 95\% CI, 1.02 to 1.45 ) (Table 3).

\section{Hyperthyroidism and stroke}

Eleven studies demonstrated the relationship between overt hyperthyroidism and stroke (Fig. 3A). The result of meta-analysis showed a significant association (HR, $1.35 ; 95 \% \mathrm{CI}, 1.03$ to $1.75)$ with moderate heterogeneity $\left(I^{2}=51 \%, P=0.02\right)$. In the subgroup analysis by age and CVD risk, significant association 


\begin{tabular}{|c|c|c|c|c|}
\hline & & Weight & Weight & Hazard Ratio \\
\hline Study & TE SE & (fixed) & (random) & IV, Fixed + Random, 95\% \\
\hline Okosieme 2019 & 0.200 .189 & $3.7 \%$ & $4.6 \%$ & $1.22[0.84 ; 1.77]$ \\
\hline Kim 2020 & 0.150 .059 & $37.0 \%$ & $38.6 \%$ & $1.16[1.03 ; 1.30]$ \\
\hline Ryodi 2018 & 0.070 .048 & $57.1 \%$ & $54.0 \%$ & $1.07[0.97 ; 1.18]$ \\
\hline Martin 2017 & 0.070 .246 & $2.2 \%$ & $2.7 \%$ & $1.07[0.66 ; 1.73]$ \\
\hline \multicolumn{2}{|l|}{ Fixed effect modle } & $100.0 \%$ & - & $1.11[1.03 ; 1.19]$ \\
\hline \multicolumn{2}{|c|}{ Random effect model } & - & $100.0 \%$ & $1.11[1.02 ; 1.20]$ \\
\hline \multicolumn{5}{|c|}{ Heterogeneity: Tau $^{2}=0.0008 ; \mathrm{Chi}^{2}=1.41, \mathrm{df}=3(\mathrm{P}=0.70) ; 1^{2}=0 \%$} \\
\hline
\end{tabular}

\begin{tabular}{|c|c|c|c|c|}
\hline & & Weight & Weight & Hazard Ratio \\
\hline Study & $\mathrm{TE}$ & $E$ (fixed) & (random) & V, Fixed + Random, 95 \\
\hline Langen 2018 & 0.460 .258 & $8 \quad 9.1 \%$ & $10.3 \%$ & $1.58[0.95 ; 2.62]$ \\
\hline Martin 2017 & 0.170 .165 & $522.3 \%$ & $15.0 \%$ & $1.18[0.85 ; 1.63]$ \\
\hline Geng 2015 & 1.600 .825 & $5 \quad 0.9 \%$ & $1.8 \%$ & $4.96[0.98 ; 25.00]$ \\
\hline rechsler 2014 & 0.290 .213 & $3 \quad 13.3 \%$ & $12.4 \%$ & $1.34[0.88 ; 2.04]$ \\
\hline svold 2012 & 0.310 .207 & $7 \quad 14.0 \%$ & $12.7 \%$ & $1.37[0.91 ; 2.06]$ \\
\hline anchen 2012 & $=0.140 .412$ & $23.6 \%$ & $5.7 \%$ & $0.87[0.39 ; 1.95]$ \\
\hline oekholdt 2010 & 0.020 .216 & $6 \quad 13.0 \%$ & $12.2 \%$ & $1.02[0.67 ; 1.56]$ \\
\hline lervasi 2007 & 0.290 .264 & $48.7 \%$ & $10.1 \%$ & $1.33[0.79 ; 2.23]$ \\
\hline Cappola 2006 & 0.040 .248 & $9.8 \%$ & $10.7 \%$ & $1.04[0.64 ; 1.69]$ \\
\hline Walsh 2005 & 0.260 .435 & $3.2 \%$ & $5.2 \%$ & $1.30[0.55 ; 3.05]$ \\
\hline Gussekloo 2004 & 0.310 .513 & $2.3 \%$ & $4.0 \%$ & $1.37[0.50 ; 3.75]$ \\
\hline xed effect modle & & $100.0 \%$ & - & $1.24[1.07 ; 1.45]$ \\
\hline indom effect model & & & $100.0 \%$ & $1.26[1.06 ; 1.49]$ \\
\hline
\end{tabular}

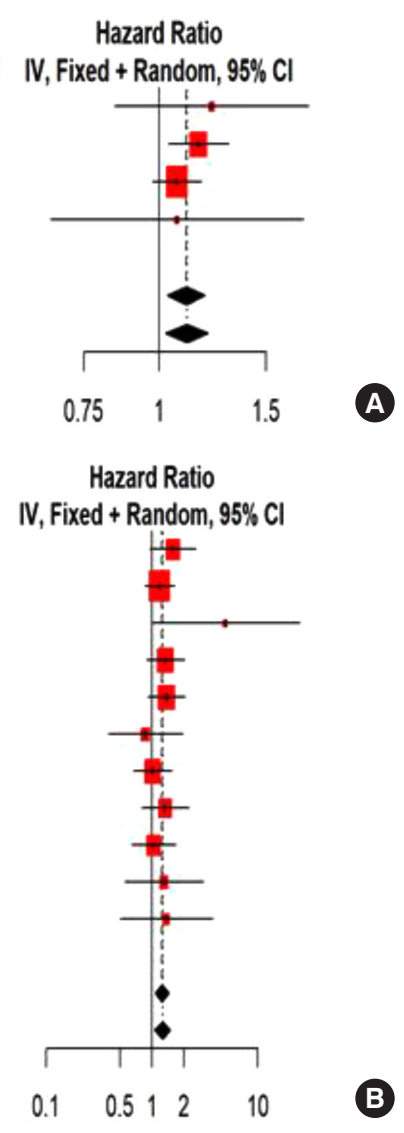

Fig. 2. Forest plots for hyperthyroidism and risk of ischemic heart disease (IHD). (A) Overt hyperthyroidism and IHD. (B) Subclinical hyperthyroidism and IHD. TE, total effect; SE, standard error; IV, inverse variance; CI, confidence interval.

between overt hyperthyroidism and stroke was particularly observed in subjects aged younger than 65 years (HR, 1.38; $95 \%$ CI, 1.06 to 1.78 ) and in those without AF (HR, 1.19; $95 \%$ CI, 1.13 to 1.25 ) (Table 2). Increased risk of stroke was consistently observed in both iodine-sufficient and -deficient areas (Supplemental Table S3).

Five studies which showed the association between subclinical hyperthyroidism and stroke were pooled (Fig. 3B). No statistically significant association was found between subclinical hyperthyroidism and stroke (HR, 1.17; 95\% CI, 0.90 to 1.52 ). Subgroup analyses did not show any relevant difference in the results (Table 2, Supplemental Table S4).

\section{Hyperthyroidism and heart failure}

Two eligible studies reported a relationship between overt hyperthyroidism and heart failure. The pooled HR for heart failure was 1.42 (95\% CI, 0.93 to 2.16$)$, with high heterogeneity $\left(I^{2}=91 \%\right.$, $P<0.01)$ (Fig. 4A).

Based on the four studies, we found no significant association between subclinical hyperthyroidism and heart failure (HR, 1.41; $95 \% \mathrm{CI}, 0.60$ to 3.30$)$ with moderated heterogeneity $\left(I^{2}=66 \%\right.$, $P=0.03$ ) (Fig. 4B).

\section{Hyperthyroidism and CV mortality}

Seven studies showed that overt hyperthyroidism is associated with increased CV mortality (HR, $1.2 ; 95 \% \mathrm{CI}, 1.00$ to 1.46 ) with high heterogeneity $\left(I^{2}=76 \%, P<0.01\right)$ (Fig. $\left.5 \mathrm{~A}\right)$. Increased risk of $\mathrm{CV}$ mortality was further observed in iodine sufficient areas by the subgroup analysis (Supplemental Table S3).

For subclinical hyperthyroidism, fourteen eligible studies were pooled to estimate the overall HR for CV mortality (Fig. 5B). No statistically significant association was found between subclinical hyperthyroidism and CV mortality (HR, 1.21; 95\% CI, 0.88 to 1.67$)$ with moderated heterogeneity $\left(I^{2}=51 \%, P=0.01\right)$. Subgroup analyses did not show any relevant difference in the results (Table 3, Supplemental Table S4). 
Table 2. Subgroup Analysis of the Association between Overt Hyperthyroidism and the Risk of Cardiovascular Events

\begin{tabular}{lccc}
\hline Subgroup & $\begin{array}{c}\text { Pooled HR } \\
(95 \% \mathrm{CI})\end{array}$ & $I^{2}, \%$ & $\begin{array}{l}\text { No. of } \\
\text { studies }\end{array}$
\end{tabular}

Ischemic heart disease

Age group

$<65 \mathrm{yr}$

$\geq 65 \mathrm{yr}$

$1.11(1.03-1.19)$

CVD risk group

High

Low

Stroke

Age group

$<65 \mathrm{yr}$

$1.38(1.06-1.78)$

54.2

10

$\geq 65 \mathrm{yr}$

$0.64(0.18-2.26)$

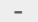

1

CVD risk

High (with A-fib)

$2.05(0.21-20.29) \quad 60.7 \quad 3$

Low

$1.19(1.13-1.25)$

35.5

8

Heart failure

Age group

$<65 \mathrm{yr}$

$\geq 65 \mathrm{yr}$

$1.42(0.93-2.16)$

90.6

CVD risk group

High

Low

NA

NA

CV mortality

Age group

$<65 \mathrm{yr}$

$1.16(0.93-1.45)$

76.4

$\geq 65 \mathrm{yr}$

$1.46(1.20-1.77)$

CVD risk group

High

Low

$1.20(1.00-1.46)$

76.3

$\mathrm{HR}$, hazard ratio; CI, confidence interval; NA, not available; CVD, cardiovascular disease; A-fib, atrial fibrillation.

\section{DISCUSSION}

In the present meta-analysis, we investigated the association of hyperthyroidism with IHD, stroke, heart failure, and CV mortality by pooling 37 cohort studies. We found that overt hyperthyroidism was associated with increased risk of IHD, stroke, and CV mortality, while subclinical hyperthyroidism was associated with increased risk of IHD.

In this study, overt hyperthyroidism seems to be associated with increased risk of CV mortality by $20 \%$ compared with the
Table 3. Subgroup Analysis of the Association between Subclinical Hyperthyroidism and the Risk of Cardiovascular Events

\begin{tabular}{lccc}
\hline Subgroup & $\begin{array}{c}\text { Pooled HR } \\
(95 \% \mathrm{CI})\end{array}$ & $I^{2}, \%$ & $\begin{array}{l}\text { No. of } \\
\text { studies }\end{array}$ \\
\hline
\end{tabular}

Ischemic heart disease

Age group

$<65 \mathrm{yr}$

$1.27(1.06-1.53)$

$\geq 65 \mathrm{yr}$

$1.17(0.88-1.55)$

$0 \quad 7$

CVD risk group

$\begin{array}{lccc}\text { High } & 1.32(0.98-1.77) & 16.7 & 4 \\ \text { Low } & 1.22(1.02-1.45) & 0 & 7\end{array}$

Stroke

Age group

$<65 \mathrm{yr} \quad 1.25(0.92-1.70) \quad 0 \quad 2$

$\geq 65 \mathrm{yr}$

$1.12(0.13-9.93)$

68.6

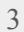

CVD risk

$\begin{array}{lrrr}\text { High } & 1.48(0.32-6.84) & 81 & 2 \\ \text { Low } & 1.16(0.87-1.55) & 0 & 3\end{array}$

Heart failure

Age group

$\begin{array}{llcc}<65 \mathrm{yr} & 1.52(0.86-2.69) & - & 1 \\ \geq 65 \mathrm{yr} & 1.40(0.27-7.34) & 75.2 & 3\end{array}$

CVD risk group

$\begin{array}{llll}\text { High } & 1.73(0.56-5.36) & 86.9 & 2 \\ \text { Low } & 1.24(0.81-1.92) & 12.9 & 2\end{array}$

CV mortality

Age group

$\begin{array}{llll}<65 \mathrm{yr} & 1.34(0.61-2.94) & 67 & 6 \\ \geq 65 \mathrm{yr} & 0.99(0.79-1.25) & 18.7 & 9\end{array}$

CVD risk group

$\begin{array}{lllr}\text { High } & 1.26(0.40-3.91) & 74.7 & 5 \\ \text { Low } & 1.26(1.00-1.59) & 14.1 & 10\end{array}$

HR, hazard ratio; CI, confidence interval; NA, not available; CVD, cardiovascular disease.

euthyroid control or general population, and increased IHD and stroke risk appear to be the cause of CV mortality. To the best of our knowledge, this is the first meta-analysis evaluating the association of overt hyperthyroidism with IHD and stroke. There are several plausible mechanisms that could explain the increased risk of atherosclerotic event in hyperthyroid patients. Excess thyroid hormone is known to be associated with endothelial damage and increased procoagulant proteins, including von Willebrand factor, fibrinogen, and factors VIII and IX [49]. Recently, Bano et al. [3] also suggested that free thyroxine levels may be 


\begin{tabular}{|c|c|c|c|c|}
\hline & & Weight & Weight & Hazard Ratio \\
\hline Study & $\mathrm{TE}$ & $E$ (fixed) & (random) & Fixed + Random, 95\% \\
\hline Kim 2020 & 0.110 .037 & $742.4 \%$ & $13.4 \%$ & $1.12[1.04 ; 1.20]$ \\
\hline Okosieme 2019 & 0.470 .186 & $6 \quad 1.6 \%$ & $11.0 \%$ & $1.60[1.11 ; 2.30]$ \\
\hline Ryodi 2018 & 0.200 .063 & $314.5 \%$ & $13.2 \%$ & $1.22[1.08 ; 1.38]$ \\
\hline Martin 2017 & 0.100 .274 & $4 \quad 0.8 \%$ & $9.0 \%$ & $1.10[0.64 ; 1.88]$ \\
\hline Yang 2015 & 0.290 .065 & $5 \quad 13.6 \%$ & $13.1 \%$ & $1.34[1.18 ; 1.52]$ \\
\hline Bruere 2015 & -0.450 .643 & $3 \quad 0.1 \%$ & $3.6 \%$ & $0.64[0.18 ; 2.26]$ \\
\hline La Cour 2015 & 0.170 .048 & $8 \quad 24.9 \%$ & $13.3 \%$ & $1.18[1.07 ; 1.30]$ \\
\hline Chen 2014 & 1.160 .436 & $6 \quad 0.3 \%$ & $6.0 \%$ & $3.20[1.36 ; 7.53]$ \\
\hline Sheu 2010 & 0.360 .187 & $1.6 \%$ & $11.0 \%$ & $1.44[1.00 ; 2.08]$ \\
\hline Siu 2009 & 1.250 .562 & $0.2 \%$ & $4.4 \%$ & $3.50[1.16 ; 10.54]$ \\
\hline Qureshi 2006 & .0 .690 .928 & $0.1 \%$ & $2.0 \%$ & $0.50[0.08 ; 3.08]$ \\
\hline Fixed effect modle & & $100.0 \%$ & -. & $1.19[1.14 ; 1.25]$ \\
\hline Random effect model & & .. & $100.0 \%$ & $1.35[1.03 ; 1.75]$ \\
\hline
\end{tabular}

Weight Weight Hazard Ratio

Study

Langen 2018

Martin 2017

TE SE (fixed) (random) IV, Fixed + Random, 95\% Cl IV, Fixed + Random, 95\% Cl

Drechsler 2014

$0.290 .344 \quad 15.1 \% \quad 21.1 \%$

$0.210 .177 \quad 57.3 \% \quad 27.9 \%$

$1.34[0.68 ; 2.63]$

$\begin{array}{rll}-0.34 & 0.401 & 11.2 \% \quad 18.8 \%\end{array}$

Schultz 2011

$1.22 \quad 0.552 \quad 5.9 \% \quad 13.8 \%$

$1.23[0.87 ; 1.74]$

$0.71[0.32 ; 1.56]$

Cappola 2006

$-0.360 .414 \quad 10.5 \% \quad 18.3 \%$

$3.39[1.15 ; 10.00]$

$0.70[0.31 ; 1.58]$

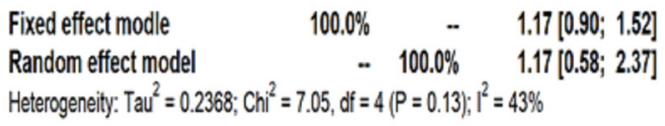

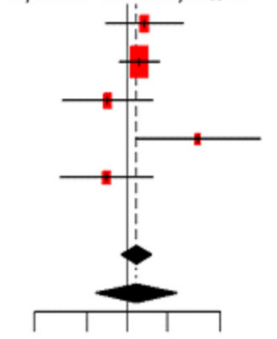

0.20 .5125

\section{A}

Fig. 3. Forest plots for hyperthyroidism with the risk of stroke. (A) Overt hyperthyroidism and stroke. (B) Subclinical hyperthyroidism and stroke. TE, total effect; SE, standard error; IV, inverse variance; CI, confidence interval.

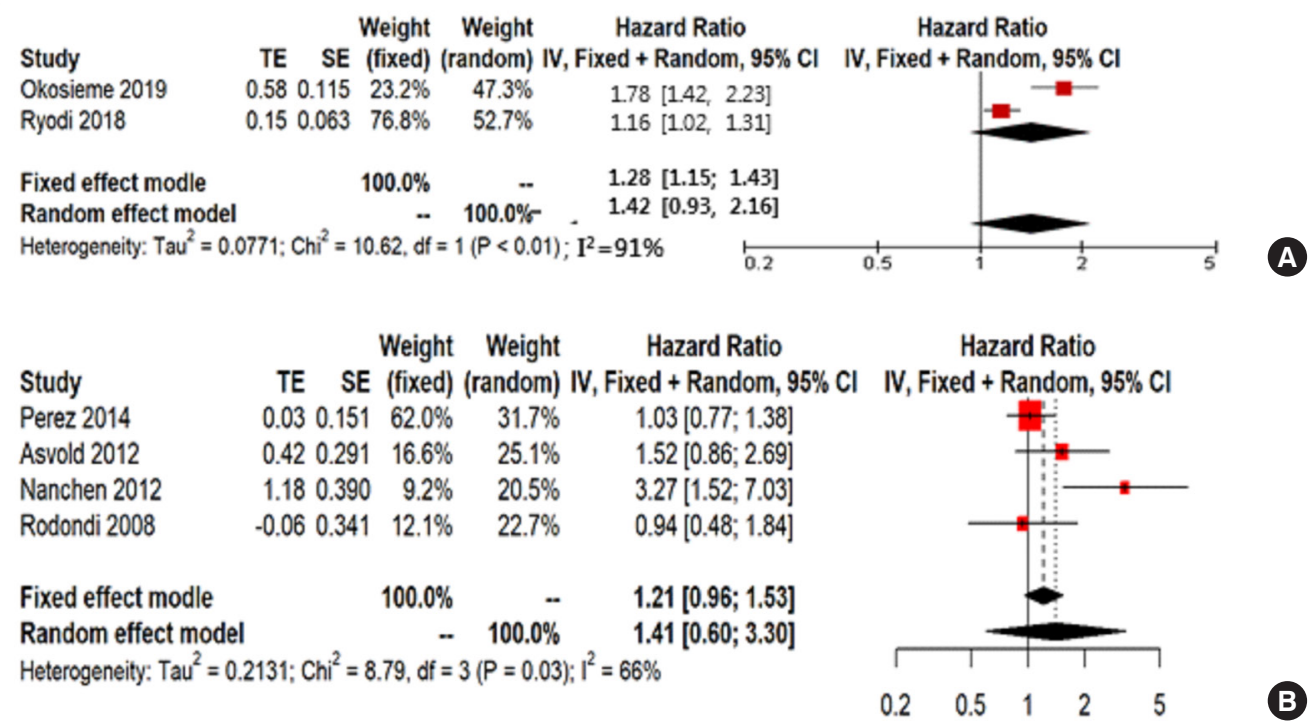

Fig. 4. Forest plots for hyperthyroidism with the risk of heart failure. (A) Overt hyperthyroidism and heart failure. (B) Subclinical hyperthyroidism and heart failure. TE, total effect; SE, standard error; IV, inverse variance; CI, confidence interval. 


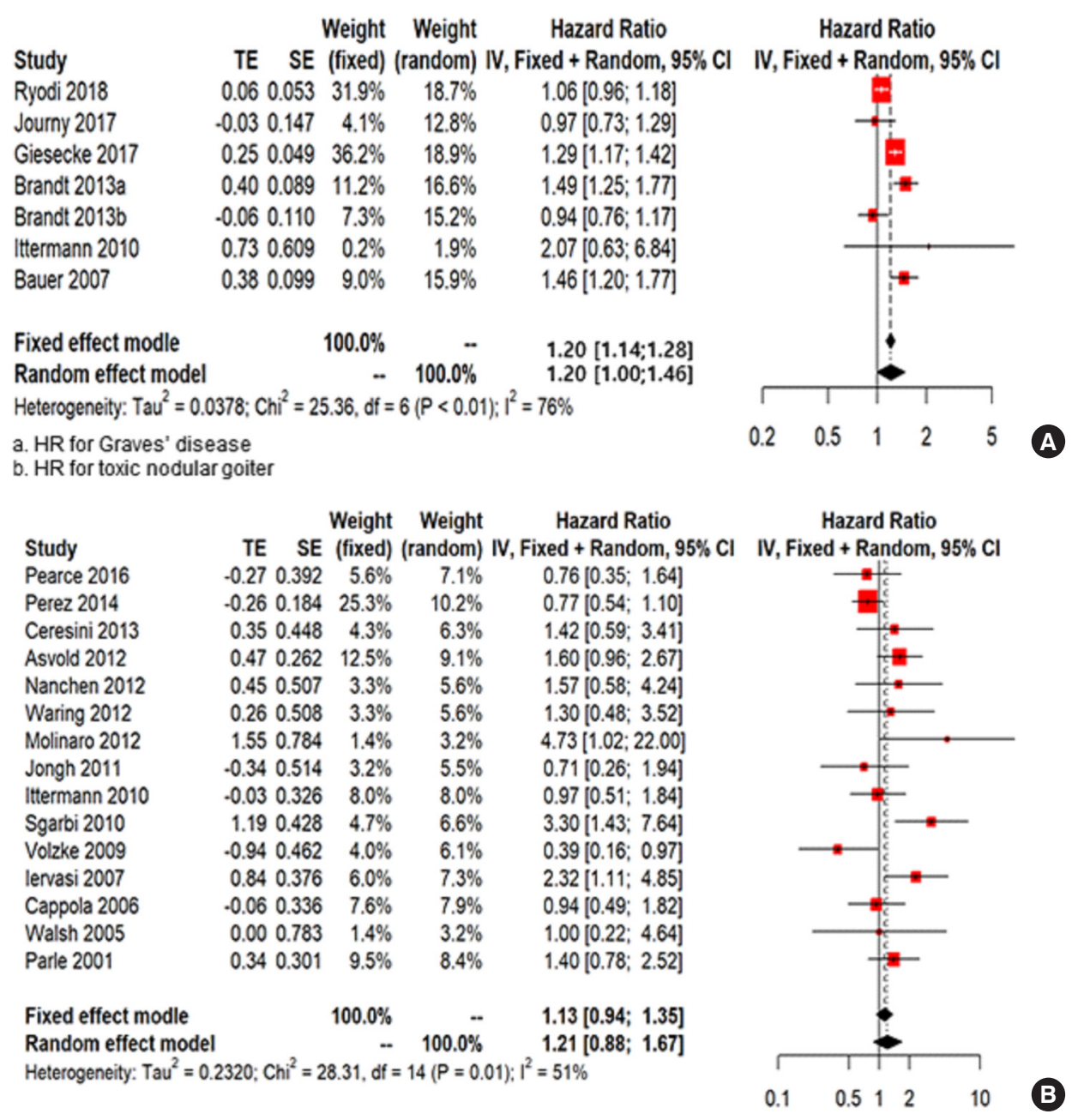

Fig. 5. Forest plots for hyperthyroidism with the risk of cardiovascular (CV) mortality. (A) Overt hyperthyroidism and CV mortality. (B) Subclinical hyperthyroidism and CV mortality. TE, total effect; SE, standard error; IV, inverse variance; CI, confidence interval.

positively associated with atherosclerotic events, independent of conventional CV risk factors. Regarding the association between hyperthyroidism and stroke, some studies have suggested that AF induced by hyperthyroidism may increase the risk of thromboembolic events, such as ischemic stroke [13]. In our subgroup analyses by CVD risk, significant association between overt hyperthyroidism and stroke was only observed in subjects without AF. Therefore, it seems unlikely that the increased risk of stroke in overt hyperthyroidism is mediated by AF; however, additional prospective cohort studies are required to confirm this finding. In the subgroup analysis by age group, increased risk of stroke was observed in hyperthyroid patients aged $<65$ years. However, a small number of studies were conducted on the hyperthyroid patients older than 65 years, it was not possible to conclude whether age-related risks were different.
Hyperthyroidism is commonly associated with hyperdynamic circulation characterized by decreased peripheral vascular resistance and increased total blood volume, heart rate, and contractility [2]. Previous studies found significant echocardiographic abnormalities, such as increased left ventricle mass, systolic, and diastolic dysfunction in hyperthyroid patients [50]. In the current meta-analysis, we did not find any association between hyperthyroidism and heart failure; however, our finding should be interpreted with caution. Because we included only cohort studies that investigated the incidence of hospitalization for heart failure in hyperthyroid patients, only a small number of studies met such criteria. Moreover, the incidence rate of reported heart failure events was likely underestimated in cohort studies since some may have developed heart failure without hospitalization.

With respect to the association between subclinical hyperthy- 
roidism and $\mathrm{CV}$ events, we found an increased risk of IHD in patients with subclinical hyperthyroidism compared to those with euthyroid control, findings are consistent with previous meta-analyses $[14,45]$. As shown in our subgroup analysis, a significant association between subclinical hyperthyroidism and IHD was only observed in those with low CVD risk. This finding suggests that subclinical hyperthyroidism may be an independent risk factor for IHD in subjects without CVD. In age stratified analysis, increased risk of IHD was found among subjects aged less than 65 years. This result is similar with recent meta-analysis by Sun et al. [45]; they showed that the risk of coronary heart disease mortality was higher in those aged less than 65 years. According to the current guideline, treatment for subclinical hyperthyroidism is recommended in all individuals over the age of 65 years, as well as in patients with heart disease, symptoms of hyperthyroidism or osteoporosis when TSH is persistently less than $0.1 \mathrm{mIU} / \mathrm{L}$ [51]. This recommendation was based primarily on the studies showing an increased rate of $\mathrm{AF}$ and $\mathrm{CV}$ mortality and altered skeletal heath in younger as well as older patients with a suppressed TSH level [14,52]. Although, there is no subgroup analysis according to the TSH level in this meta-analysis, our findings suggest that a careful follow-up strategy for CVD is warranted in subjects with subclinical hyperthyroidism, even if they are less than the age of 65 years or have no underlying CVD. Large prospective studies are needed to clarify the benefit of treating low risk younger patients with subclinical hyperthyroidism.

In the current meta-analysis, we did not find any association between subclinical hyperthyroidism and stroke, which is in line with a previous meta-analysis [12]. Although subclinical hyperthyroidism significantly increases the risk of AF, as demonstrated in a large cohort study [4], the risk of stroke in subclinical hyperthyroidism remains unclear based on current evidence. We also found no significant association between subclinical hyperthyroidism and heart failure/CV mortality. This result is different from previous meta-analysis, which reported increased risk of heart failure and CV mortality, especially when TSH level is lower than $0.1 \mathrm{mIU} / \mathrm{L}[14,19]$. First, this might be due to the lack of subgroup analyses based on different TSH levels in this meta-analysis. Only one study in our meta-analysis analyzed the association between risk of heart failure incidence and degree of subclinical hyperthyroidism. Second, the difference may be due to the recent cohort studies added in the current analysis [5,31,33,41].

In the subgroup analyses by regional iodine intake status, an increased risk of IHD, stroke and CV mortality in patients with overt hyperthyroidism was generally observed in iodine-sufficient areas. Since Graves' disease is the most prevalent cause of hyperthyroidism in iodine-sufficient areas, this finding suggested that thyroid autoimmunity might be associated with an increased risk of CVD. Further studies based on individual participant data are needed to confirm this finding.

There are several strengths in our study. First, we analyzed the association of both overt and subclinical hyperthyroidism with specific CV events, including IHD, stroke, HF, and CV mortality, instead of combining these events into a single combined CV event. Second, we included many cohort studies with a large population and conducted subgroup analyses for various outcome measures. However, our study also has some limitations. First, this study lacked results of individual thyroid function tests, and thus, we were unable to perform a subgroup analysis according to different TSH levels. Second, although previous study has suggested that radioiodine treatment might be associated with increased CV risk in hyperthyroid patients [27], association with treatment method was not evaluated in this meta-analysis because very few studies included this parameter. Third, for the association of overt hyperthyroidism with IHD and heart failure, subgroup analysis according to age and CVD risk could not be performed since only a small number of studies met the inclusion criteria.

In conclusion, our meta-analysis showed that overt hyperthyroidism is associated with increased risk of IHD, stroke, and CV mortality, while subclinical hyperthyroidism is associated with increased risk of IHD. The associations outlined in the present study were more likely to be significant in subjects without underlying CVD. Future studies based on individual participant data are needed to better determine the association between hyperthyroidism and risk of $\mathrm{CV}$ events.

\section{CONFLICTS OF INTEREST}

No potential conflict of interest relevant to this article was reported.

\section{ACKNOWLEDGMENTS}

This study was supported by the Korean Endocrine Society of EnM Research Award 2018.

\section{AUTHOR CONTRIBUTIONS}

Conception or design: S.Y.S., M.K.L. Acquisition, analysis, or 
interpretation of data: S.Y.S., E.L. Drafting the work or revising: S.Y.S., E.L. Final approval of the manuscript: S.Y.S., J.H.L.

\section{ORCID}

Seo Young Sohn https://orcid.org/0000-0003-2033-6671

\section{REFERENCES}

1. Kwon H, Jung JH, Han KD, Park YG, Cho JH, Lee DY, et al. Prevalence and annual incidence of thyroid disease in Korea from 2006 to 2015: a nationwide population-based cohort study. Endocrinol Metab (Seoul) 2018;33:260-7.

2. Jabbar A, Pingitore A, Pearce SH, Zaman A, Iervasi G, Razvi S. Thyroid hormones and cardiovascular disease. Nat Rev Cardiol 2017;14:39-55.

3. Bano A, Chaker L, Mattace-Raso FUS, van der Lugt A, Ikram MA, Franco OH, et al. Thyroid function and the risk of atherosclerotic cardiovascular morbidity and mortality: the Rotterdam Study. Circ Res 2017;121:1392-400.

4. Selmer C, Olesen JB, Hansen ML, Lindhardsen J, Olsen AM, Madsen JC, et al. The spectrum of thyroid disease and risk of new onset atrial fibrillation: a large population cohort study. BMJ 2012;345:e7895.

5. Asvold BO, Bjoro T, Platou C, Vatten LJ. Thyroid function and the risk of coronary heart disease: 12-year follow-up of the HUNT study in Norway. Clin Endocrinol (Oxf) 2012;77: 911-7.

6. Bauer DC, Rodondi N, Stone KL, Hillier TA; Study of Osteoporotic Fractures Research Group: Universities of California (San Francisco), Pittsburgh, Minnesota (Minneapolis); Kaiser Permanente Center for Health Research, Portland. Thyroid hormone use, hyperthyroidism and mortality in older women. Am J Med 2007;120:343-9.

7. Boekholdt SM, Titan SM, Wiersinga WM, Chatterjee K, Basart DC, Luben R, et al. Initial thyroid status and cardiovascular risk factors: the EPIC-Norfolk prospective population study. Clin Endocrinol (Oxf) 2010;72:404-10.

8. Brandt F, Thvilum M, Almind D, Christensen K, Green A, Hegedus L, et al. Graves' disease and toxic nodular goiter are both associated with increased mortality but differ with respect to the cause of death: a Danish population-based register study. Thyroid 2013;23:408-13.

9. Bruere H, Fauchier L, Bernard Brunet A, Pierre B, Simeon E, Babuty D, et al. History of thyroid disorders in relation to clinical outcomes in atrial fibrillation. Am J Med 2015;128:
30-7.

10. Cappola AR, Fried LP, Arnold AM, Danese MD, Kuller LH, Burke GL, et al. Thyroid status, cardiovascular risk, and mortality in older adults. JAMA 2006;295:1033-41.

11. Ceresini G, Ceda GP, Lauretani F, Maggio M, Usberti E, Marina M, et al. Thyroid status and 6-year mortality in elderly people living in a mildly iodine-deficient area: the aging in the Chianti Area Study. J Am Geriatr Soc 2013;61: 868-74.

12. Chaker L, Baumgartner C, Ikram MA, Dehghan A, Medici $\mathrm{M}$, Visser WE, et al. Subclinical thyroid dysfunction and the risk of stroke: a systematic review and meta-analysis. Eur J Epidemiol 2014;29:791-800.

13. Chen Q, Yan Y, Zhang L, Cheng K, Liu Y, Zhu W. Effect of hyperthyroidism on the hypercoagulable state and thromboembolic events in patients with atrial fibrillation. Cardiology 2014;127:176-82.

14. Collet TH, Gussekloo J, Bauer DC, den Elzen WP, Cappola AR, Balmer P, et al. Subclinical hyperthyroidism and the risk of coronary heart disease and mortality. Arch Intern Med 2012;172:799-809.

15. de Jongh RT, Lips P, van Schoor NM, Rijs KJ, Deeg DJ, Comijs HC, et al. Endogenous subclinical thyroid disorders, physical and cognitive function, depression, and mortality in older individuals. Eur J Endocrinol 2011;165:545-54.

16. Drechsler C, Schneider A, Gutjahr-Lengsfeld L, Kroiss M, Carrero JJ, Krane V, et al. Thyroid function, cardiovascular events, and mortality in diabetic hemodialysis patients. Am J Kidney Dis 2014;63:988-96.

17. Ryodi E, Metso S, Huhtala H, Valimaki M, Auvinen A, Jaatinen P. Cardiovascular morbidity and mortality after treatment of hyperthyroidism with either radioactive iodine or thyroidectomy. Thyroid 2018;28:1111-20.

18. Franklyn JA, Maisonneuve P, Sheppard MC, Betteridge J, Boyle P. Mortality after the treatment of hyperthyroidism with radioactive iodine. N Engl J Med 1998;338:712-8.

19. Gencer B, Collet TH, Virgini V, Bauer DC, Gussekloo J, Cappola AR, et al. Subclinical thyroid dysfunction and the risk of heart failure events: an individual participant data analysis from 6 prospective cohorts. Circulation 2012;126: 1040-9.

20. Geng J, Lu W, Hu T, Tao S, Zhang H, Chen J, et al. Subclinical hyperthyroidism increases risk of coronary heart disease events in type 2 diabetes mellitus. Endocrine 2015;49:557-9.

21. Giesecke P, Rosenqvist M, Frykman V, Friberg L, Wallin G, Hoijer J, et al. Increased cardiovascular mortality and mor- 
bidity in patients treated for toxic nodular goiter compared to Graves' disease and nontoxic goiter. Thyroid 2017;27:87885.

22. Gussekloo J, van Exel E, de Craen AJ, Meinders AE, Frolich M, Westendorp RG. Thyroid status, disability and cognitive function, and survival in old age. JAMA 2004;292:2591-9.

23. Iervasi G, Molinaro S, Landi P, Taddei MC, Galli E, Mariani F, et al. Association between increased mortality and mild thyroid dysfunction in cardiac patients. Arch Intern Med 2007;167:1526-32.

24. Ittermann T, Haring R, Sauer S, Wallaschofski H, Dorr M, Nauck M, et al. Decreased serum TSH levels are not associated with mortality in the adult northeast German population. Eur J Endocrinol 2010;162:579-85.

25. Journy NMY, Bernier MO, Doody MM, Alexander BH, Linet MS, Kitahara CM. Hyperthyroidism, hypothyroidism, and cause-specific mortality in a large cohort of women. Thyroid 2017;27:1001-10.

26. Kim HJ, Kang T, Kang MJ, Ahn HS, Sohn SY. Incidence and mortality of myocardial infarction and stroke in patients with hyperthyroidism: a nationwide cohort study in Korea. Thyroid 2020;30:955-65.

27. la Cour JL, Jensen LT, Vej-Hansen A, Nygaard B. Radioiodine therapy increases the risk of cerebrovascular events in hyperthyroid and euthyroid patients. Eur J Endocrinol 2015; 172:771-8.

28. Langen VL, Niiranen TJ, Puukka P, Lehtonen AO, Hernesniemi JA, Sundvall J, et al. Thyroid-stimulating hormone and risk of sudden cardiac death, total mortality and cardiovascular morbidity. Clin Endocrinol (Oxf) 2018;88:105-13.

29. Martin SS, Daya N, Lutsey PL, Matsushita K, Fretz A, McEvoy JW, et al. Thyroid function, cardiovascular risk factors, and incident atherosclerotic cardiovascular disease: the Atherosclerosis Risk in Communities (ARIC) Study. J Clin Endocrinol Metab 2017;102:3306-15.

30. Molinaro S, Iervasi G, Lorenzoni V, Coceani M, Landi P, Srebot V, et al. Persistence of mortality risk in patients with acute cardiac diseases and mild thyroid dysfunction. Am J Med Sci 2012;343:65-70.

31. Nanchen D, Gussekloo J, Westendorp RG, Stott DJ, Jukema JW, Trompet S, et al. Subclinical thyroid dysfunction and the risk of heart failure in older persons at high cardiovascular risk. J Clin Endocrinol Metab 2012;97:852-61.

32. Okosieme OE, Taylor PN, Evans C, Thayer D, Chai A, Khan I, et al. Primary therapy of Graves' disease and cardiovascular morbidity and mortality: a linked-record cohort study. Lancet Diabetes Endocrinol 2019;7:278-87.

33. Pearce SH, Razvi S, Yadegarfar ME, Martin-Ruiz C, Kingston A, Collerton J, et al. Serum thyroid function, mortality and disability in advanced old age: the Newcastle 85+ Study. J Clin Endocrinol Metab 2016;101:4385-94.

34. Perez AC, Jhund PS, Stott DJ, Gullestad L, Cleland JG, van Veldhuisen DJ, et al. Thyroid-stimulating hormone and clinical outcomes: the CORONA trial (controlled rosuvastatin multinational study in heart failure). JACC Heart Fail 2014;2: $35-40$.

35. Rodondi N, Bauer DC, Cappola AR, Cornuz J, Robbins J, Fried LP, et al. Subclinical thyroid dysfunction, cardiac function, and the risk of heart failure. The Cardiovascular Health study. J Am Coll Cardiol 2008;52:1152-9.

36. Schultz M, Kistorp C, Raymond I, Dimsits J, Tuxen C, Hildebrandt $\mathrm{P}$, et al. Cardiovascular events in thyroid disease: a population based, prospective study. Horm Metab Res 2011; 43:653-9.

37. Sgarbi JA, Matsumura LK, Kasamatsu TS, Ferreira SR, Maciel RM. Subclinical thyroid dysfunctions are independent risk factors for mortality in a 7.5-year follow-up: the Japanese-Brazilian thyroid study. Eur J Endocrinol 2010; 162:569-77.

38. Sheu JJ, Kang JH, Lin HC, Lin HC. Hyperthyroidism and risk of ischemic stroke in young adults: a 5-year follow-up study. Stroke 2010;41:961-6.

39. Siu CW, Pong V, Zhang X, Chan YH, Jim MH, Liu S, et al. Risk of ischemic stroke after new-onset atrial fibrillation in patients with hyperthyroidism. Heart Rhythm 2009;6:16973.

40. Volzke H, Menzel D, Henzler J, Robinson D, Motz W, Rettig R, et al. Serum thyrotropin levels predict all-cause and circulatory mortality in patients with invasively treated coronary artery disease. Int J Cardiol 2009;133:407-10.

41. Waring AC, Harrison S, Samuels MH, Ensrud KE, LeBLanc ES, Hoffman AR, et al. Thyroid function and mortality in older men: a prospective study. J Clin Endocrinol Metab 2012; 97:862-70.

42. Yang MH, Yang FY, Lee DD. Thyroid disease as a risk factor for cerebrovascular disease. J Stroke Cerebrovasc Dis 2015;24:912-20.

43. Parle JV, Maisonneuve P, Sheppard MC, Boyle P, Franklyn JA. Prediction of all-cause and cardiovascular mortality in elderly people from one low serum thyrotropin result: a 10year cohort study. Lancet 2001;358:861-5.

44. Qureshi AI, Suri FK, Nasar A, Kirmani JF, Divani AA, Giles 
WH. Free thyroxine index and risk of stroke: results from the National Health and Nutrition Examination Survey Follow-up Study. Med Sci Monit 2006;12:CR501-6.

45. Sun J, Yao L, Fang Y, Yang R, Chen Y, Yang K, et al. Relationship between subclinical thyroid dysfunction and the risk of cardiovascular outcomes: a systematic review and meta-analysis of prospective cohort studies. Int J Endocrinol 2017;2017:8130796.

46. Walsh JP, Bremner AP, Bulsara MK, O'Leary P, Leedman PJ, Feddema P, et al. Subclinical thyroid dysfunction as a risk factor for cardiovascular disease. Arch Intern Med 2005; 165:2467-72.

47. OpenGrey. System for information on Grey literature in Europe [Internet]. OpenGrey; 2020 [cited 2020 Oct 8]. Available from: http://www.opengrey.eu/.

48. Zimmermann MB, Andersson M. Update on iodine status worldwide. Curr Opin Endocrinol Diabetes Obes 2012;19:
382-7.

49. Shih CH, Chen SL, Yen CC, Huang YH, Chen CD, Lee YS, et al. Thyroid hormone receptor-dependent transcriptional regulation of fibrinogen and coagulation proteins. Endocrinology 2004;145:2804-14.

50. Biondi B, Palmieri EA, Fazio S, Cosco C, Nocera M, Sacca L, et al. Endogenous subclinical hyperthyroidism affects quality of life and cardiac morphology and function in young and middle-aged patients. J Clin Endocrinol Metab 2000;85:4701-5.

51. Ross DS, Burch HB, Cooper DS, Greenlee MC, Laurberg P, Maia AL, et al. 2016 American Thyroid Association guidelines for diagnosis and management of hyperthyroidism and other causes of thyrotoxicosis. Thyroid 2016;26:1343-421.

52. Blum MR, Bauer DC, Collet TH, Fink HA, Cappola AR, da Costa BR, et al. Subclinical thyroid dysfunction and fracture risk: a meta-analysis. JAMA 2015;313:2055-65. 


\section{Appendix 1. Study protocol}

1. Title: The association of overt and subclinical hyperthyroidism with the risk of cardiovascular events and cardiovascular mortality: Meta-analysis and systematic review of cohort studies.

2. Objectives: This study is conducted to evaluate the association of overt and subclinical hyperthyroidism with the risk of ischemic heart disease (IHD), stroke, heart failure, and cardiovascular mortality.

3. Protocol and registration: Methods of database search, study selection, data extraction, assessment of study quality and risk of bias, and statistical analysis are predefined in the protocol at the beginning of the study.

4. Reporting: This meta-analysis and systematic review was reported according to the Preferred Reporting Items for Systematic Review and Meta-Analysis (PRISMA) statement.

\section{Eligible criteria}

1) Study characteristics

(1) Population: patients with hyperthyroidism

(2) Intervention: none

(3) Comparison: euthyroid population or general population without hyperthyroidism

(4) Outcomes of interests

(1) IHDs

(2) Stroke

(3) Hospitalization for heart failure

(4) Cardiovascular mortality

(5) Study design: prospective or retrospective cohort studies

2) Report characteristics

(1) Years considered: published from inception to February 2020

(2) Language: English

(3) Publication status: full-text articles without limitation of publication status

3) Inclusion and exclusion criteria

(1) We only included cohort studies investigating the relationship of hyperthyroidism with cardiovascular events.

(2) We included cohort studies reporting at least one cardiovascular outcome including ischemic heart disease, stroke, heart failure, and cardiovascular mortality.

(3) We included cohort studies reporting cardiovascular outcome as hazard ratio (HR) with 95\% confidence interval (CI).

(4) We included cohort studies comparing the endogenous hyperthyroidism between euthyroid control group and the general population without hyperthyroidism.

(5) In case of duplicates or extensions, we only included a study with the longer duration or more information.

(6) We included full-text articles with no restriction of publication status.

(7) We excluded studies evaluating the effect of exogenous hyperthyroidism

6. Information sources: We searched electronic databases of MEDLINE, Embase, and the website of OpenGrey.

7. Search strategy: Cohort studies regarding the association between hyperthyroidism and cardiovascular events were searched using following criteria.

1) MEDLINE

\#1. "Thyroid diseases" OR "hyperthyroidism" OR "thyroid function tests" OR "thyrotropin" OR "thyroid hormones"

\#2. "Myocardial ischemia" OR "heart failure" OR "stroke" OR "brain ischemia” OR "mortality" OR "death"

\#3. \#1 AND \#2

\#4. "Hyperthyroidism" OR "thyroid dysfunction" OR "thyrotoxicosis" OR "Graves' disease" OR "thyroid function" OR "thyroid diseases"

\#5. "Ischemic heart disease" OR "coronary heart disease" OR "coronary disease" OR "myocardial infarction" OR "stroke" OR "heart failure" OR "death" OR "mortality" OR "cardiovascular" 
\#6. \#4 AND \#5

2) EMBASE

\#1. 'Hyperthyroidism' OR 'thyroid dysfunction' OR ‘thyrotoxicosis' OR 'Graves' disease' OR 'thyroid function' OR 'thyroid diseases'

\#2. 'Ischemic heart disease' OR ‘coronary heart disease' OR ‘coronary disease' OR 'myocardial infarction' OR 'stroke' OR 'heart failure' OR 'death' OR 'mortality' OR 'cardiovascular'

\#3. \#1 AND \#2

3) Website of OpenGrey

\#1. "Hyperthyroidism" OR "thyroid dysfunction" OR "thyrotoxicosis" OR "thyroid function" OR "thyroid diseases"

\#2. "Ischemic heart disease" OR "coronary heart disease" OR "coronary disease" OR "myocardial infarction" OR "stroke"

OR "heart failure" OR “death" OR "mortality" OR "cardiovascular"

\#3. \#1 AND \#2

8. Study selection: All identified records were evaluated for eligibility by two reviewers independently. We reviewed titles, abstracts, and full texts of the studies thoroughly. Any disagreements were resolved by a third reviewer.

9. Data extraction: Standardized data extraction was performed by two reviewers independently as follows. Any disagreements were resolved by a third reviewer.

1) First author

2) Publication year

3) Country

4) Number of study participants

5) Characteristics of study participants: mean or median age, classification of hyperthyroidism, underlying comorbidity including history of coronary, cerebral, or peripheral artery disease; heart failure; atrial fibrillation; diabetes mellitus; or chronic kidney disease and regional iodine intake (sufficient area vs. deficient area)

6) Cardiovascular outcome explored

7) HRs for each cardiovascular outcome

10. Assessment of study quality and risk bias: We assessed quality and risk of bias of included studies using the Newcastle-Ottawa Scale. Two reviewers independently evaluated each study based on following aspects of trials:

1) Selection: representativeness of the exposed cohort, selection of the non-exposed cohort, ascertainment of hyperthyroidism, demonstration that outcome of interest was not present at start of study

2) Comparability: comparability of cohorts based on the design or analysis

3) Outcome: assessment of outcome, adequacy of follow-up of cohorts

\section{Data synthesis}

1) Statistical analysis: We calculated pooled HRs with $95 \%$ CI for each cardiovascular outcome. We used both fixed and randomeffects model to evaluate pooled HR.

2) Subgroup analysis: We performed subgroup analyses by age ( $<65$ years, $\geq 65$ years), CVD risk and regional iodine intake status (sufficient area vs. deficient area).

3 ) Identifying and measuring statistical heterogeneity: We used $I^{2}$ statistic for measuring the degree of heterogeneity. 